\title{
Low incidence of pulmonary complications following nonmyeloablative stem cell transplantation
}

\author{
S. Nusair*, R. Breuer*, M.Y. Shapira*, N. Berkman*, R. Or
}

Low incidence of pulmonary complications following nonmyeloablative stem cell transplantation. S. Nusair, R. Breuer, M.Y. Shapira, N. Berkman, R. Or. (C)ERS Journals Ltd 2004.

ABSTRACT: Bone marrow transplantation is associated with pulmonary opportunistic infections and immune-mediated pulmonary processes such as idiopathic pneumonia syndrome and bronchiolitis obliterans. The aim of the present study was to test the hypothesis that nonmyeloablative stem cell transplantation (NST) has less adverse effects on the lungs.

A review was undertaken of the pulmonary complications occurring in 53 patients with various haematological malignancies, some of whom were considered high-risk patients with chemoresistant disease, who underwent fludarabine-based irradiation-free conditioning for NST performed between March 1996 and October 1998. All data related to transplant procedure, disease outcome, graft-versus-host disease (GVHD), chest imaging, microbial cultures and lung biopsies, were retrieved from information collected prospectively at the time of transplantation.

The median follow-up period after transplantation was 45 months, with 35 patients surviving $>100$ days. Approximately half of the patients displayed some form of GVHD, with $11 \%$ developing severe chronic GVHD. In $17(32 \%)$ patients, the lungs were somehow adversely affected. Only two $(3.8 \%)$ patients developed a clinical picture consistent with idiopathic pneumonia syndrome and none developed diffuse alveolar haemorrhage or bronchiolitis obliterans.

Dose-reduced conditioning is associated with a low rate of pulmonary toxicity and side-effects. These findings may extend understanding of significant immune-mediated complications occurring after bone marrow transplantation.

Eur Respir J 2004; 23: 440-445.
*Institute of Pulmonology, and ${ }^{\#}$ Dept of Bone Marrow Transplantation, Hadassah University Hospital and Hebrew University-Hadassah School of Medicine, Jerusalem, Israel.

Correspondence: S. Nusair

Institute of Pulmonology

Hadassah University Hospital

P.O. Box 12072

91120 Jerusalem

Israel

Fax: 97226435897

E-mail: samjack@shani.net

Keywords: Bronchiolitis obliterans diffuse alveolar haemorrhage idiopathic pneumonia syndrome nonmyeloablative

stem cell transplantation

Received: May 132003

Accepted after revision: November 62003
Allogeneic bone marrow transplantation (BMT) has been consistently associated with a high incidence of pulmonary complications, such as opportunistic infections caused by viruses and invasive fungi, toxic effects of chemoradiotherapy, and inflammatory processes largely associated with graft-versus-host disease (GVHD).

A devastating complication of BMT is the appearance of interstitial pneumonitis, manifested by diffuse bilateral interstitial pulmonary infiltrates associated with progressive dyspnoea and hypoxaemia. The reported incidence of interstitial pneumonitis after allogeneic BMT may be as high as $20 \%[1,2]$. In the absence of evidence of an infectious agent such as Pneumocystis carinii or cytomegalovirus (CMV), this complication is designated idiopathic pneumonia syndrome (IPS). Since the methods and sensitivity of diagnosis of specific aetiological infectious agents such as CMV differ between various reports, the true incidence of IPS after allogeneic BMT is difficult to determine but ranges from $20 \%$ in patients who have undergone conditioning protocols that include total body irradiation [1], down to $7.6 \%$ in patients for whom irradiation was not included in the conditioning protocol [3]. The higher incidence of IPS after allogeneic BMT suggests that GVHD-related immune reactions may play an important role in the pathogenesis of this condition.

For editorial comments see page 357.
The recently described nonmyeloablative conditioning, also referred to as dose-reduced conditioning, entails less intense conditioning, such that host marrow cells are not completely eradicated prior to stem cell transplantation [4]. Presumably, the subsequent elimination of malignant cells is achieved by a "graft-versus-leukemia" effect, rather than by total ablation of the host bone marrow. This method of conditioning avoids the prolonged pancytopenia and profound immune suppression observed with conventional myeloablative conditioning.

In the present retrospective study, the incidence and severity of IPS and other marrow transplant-related pulmonary complications were examined in patients undergoing nonmyeloablative stem cell transplantation (NST). It was hypothesised that the irradiation-free nonmyeloablative conditioning protocol would be associated with a relatively low incidence of pulmonary infections and immune-mediated pulmonary complications.

\section{Methods}

\section{Patients}

Pulmonary complications were reviewed in 53 consecutive patients who had undergone stem cell transplantation after nonmyeloablative conditioning between March 1996 and 
October 1998. Patients were included in this protocol in an attempt to accomplish malignancy eradication without myeloablative conditioning. Some of the patients in this cohort were very high-risk patients, who would have been excluded from conventional myeloablative allogeneic BMT because of chemoresistance, advanced age and multiple previous treatment modalities. Two patients with Hodgkin's lymphoma and one with non-Hodgkin's lymphoma received mediastinal irradiation therapy prior to transplantation. Three patients with lymphoma underwent autologous stem cell transplantation prior to the present procedure (two, one of whom had previously had Hodgkin's lymphoma, for nonHodgkin's lymphoma and one for Hodgkin's lymphoma). All patients consented to participate in the clinical trial, which was approved by the institutional review board of Hadassah University Hospital.

All data were retrieved from information collected prospectively at the time of transplantation and reviewed by physicians from the Dept of Bone Marrow Transplantation (Hadassah University Hospital and Hebrew UniversityHadassah School of Medicine, Jerusalem, Israel). Pulmonology consultants reviewed the results and reports of chest imaging, fibreoptic bronchoscopy, microbial culture and lung biopsy.

The underlying diseases in the patients who underwent NST are detailed in table 1 . Stem cells were obtained from fully matched human leukocyte antigen (HLA) class I and II siblings in 49 patients and mismatched from other family members in the remaining four (parents in two, son in one and cousin in one).

\section{Transplant procedure}

Granulocyte colony-stimulating factor-mobilised donor blood stem cells were collected once, after 5 days of administration of granulocyte colony-stimulating factor (GNeupogen; Hoffmann-LaRoche, Basle, Switzerland; $5 \mu \mathrm{g} \cdot \mathrm{kg}$ body weight ${ }^{-1}$ twice daily).

The conditioning protocol used in all patients has been described previously [4]. Briefly, the immunosuppressive treatment consisted of fludarabine $30 \mathrm{mg} \cdot \mathrm{m}^{-2} \cdot \mathrm{day}^{-1}$ for six consecutive days (days $-10--5$ ), oral busulfan $4 \mathrm{mg} \cdot \mathrm{kg}$ body weight $^{-1} \cdot$ day $^{-1}$ in four divided daily doses for two consecutive days (days -6 and -5) and anti-T-lymphocyte globulin (ATGFresenius AG, Munich, Germany) $10 \mathrm{mg} \cdot \mathrm{kg}$ body weight ${ }^{-1}$. $\mathrm{day}^{-1}$ for four consecutive days (days -4-1). None of the patients received haematopoietic growth factors after transplantation to accelerate neutrophil count recovery.

Prophylaxis against GVHD included intravenous cyclosporin $1.5 \mathrm{mg} \cdot \mathrm{kg}$ body weight ${ }^{-1}$ twice daily, starting on day -1 , and switching to an oral dose of $3 \mathrm{mg} \cdot \mathrm{kg}$ body weight ${ }^{-1}$ twice daily as soon as the patients were no longer receiving intravenous therapy, with the dosage tapered during the second or

Table 1.-Indication for nonmyeloablative stem cell transplantation in 53 patients with haematological malignancies

Indication

Patients $n$

Non-Hodgkin's lymphoma

Hodgkin's lymphoma

2

Acute nonlymphocytic leukaemia

Acute lymphocytic leukaemia

Chronic myelogenous leukaemia

Myelodysplastic syndrome

Multiple myeloma

Total third month after transplantation, according to their chimeric status and evidence of GVHD.

\section{Donor lymphocyte transfusions}

In order to augment the graft-versus-tumour effect, 11 patients with evidence of either residual disease or mixed chimerism in the absence of GVHD were treated with donor lymphocyte transfusions as previously described [4].

\section{Graft-versus-host disease}

Acute GVHD was graded by noting the presence of cutaneous, intestinal and/or hepatic involvement, according to International Bone Marrow Transplantation Registry severity indices [5]. Immediately upon appearance of signs and symptoms of GVHD, intravenous methylprednisolone $\left(2 \mathrm{mg} \cdot \mathrm{kg}\right.$ body weight ${ }^{-1}$ ) and cyclosporin were administered.

Chronic GVHD was recorded as new onset or progression from acute GVHD, and graded as limited (localised cutaneous or hepatic dysfunction) or extensive, if there was histological evidence of either chronic aggressive hepatitis or ocular or oral involvement.

\section{Infection prophylaxis}

Prophylaxis against $P$. carinii pneumonitis was provided by trimethoprim/sulfamethoxazole $\left(10 \mathrm{mg} \cdot \mathrm{kg}\right.$ body weight ${ }^{-1}$. day $^{-1}$ trimethoprim), administered prior to transplantation (days -10--1), and later resumed twice weekly as soon as absolute neutrophil counts were $>0.75 \times 10^{9}$ cells $\cdot \mathrm{L}^{-1}$. Prophylaxis against herpes simplex virus was provided by low-dose oral aciclovir $500 \mathrm{mg} \cdot \mathrm{m}^{-2}$ three times daily (days $-6-100$ ).

Pre-emptive therapy against CMV was guided by the results of polymerase chain reaction amplification of CMV deoxyribonucleic acid (DNA), with weekly testing starting at day -10 . If patients with initially negative test results gave positive results in two consecutive tests, therapy with aciclovir was replaced by ganciclovir at a daily dose of $5 \mathrm{mg} \cdot \mathrm{kg}$ body weight $^{-1}$ administered three times weekly. Therapy with ganciclovir was continued until at least two consequent negative test results were obtained.

\section{Procedure outcome}

Data regarding underlying disease outcome, marrow engraftment, GVHD (grade and organs involved), infectious complications, drug toxicity and pulmonary adverse effects were retrieved from patient charts.

\section{Pulmonary complications}

All patients underwent full pulmonary function testing prior to transplantation. After transplantation, repeat tests, including spirometry and determination of lung volumes, carbon monoxide transfer factor and arterial blood gas levels were performed whenever patients developed respiratory symptoms (e.g. cough and dyspnoea). Plain-film chest radiography and computed tomography were performed prior to the procedure and were repeated whenever clinically indicated. Pulmonary complications typically related to BMT were identified according to previously described criteria $[1,6]$. 
Pneumonia is diagnosed clinically on the basis of the presence of localised segmental or lobar pulmonary infiltrates on chest imaging accompanied by any one or combination of dyspnoea, fever and cough, with or without sputum production.

Pulmonary oedema after BMT is diagnosed on the basis of rapid onset of dyspnoea and hypoxaemia (often at 2-3 weeks after BMT), accompanied by weight gain and bibasilar crackles on physical examination. Chest radiographic examination reveals vascular redistribution and diffusely increased interstitial markings.

IPS is defined by the presence of diffuse nonlobar radiographic infiltrates accompanied by signs and symptoms of pneumonia and physiological changes such as hypoxaemia and an increasing alveolar to arterial oxygen gradient, and bronchoalveolar lavage (BAL) examination results that do not reveal a possible infectious agent. Findings from histological specimens, when obtained, range from interstitial mononuclear infiltrates and oedema to diffuse alveolar damage with alveolar epithelial necrosis, intra-alveolar hyaline membranes and type 2 alveolar cell hyperplasia.

Diffuse alveolar haemorrhage (DAH) is characterised by a sudden onset of dyspnoea and hypoxaemia, accompanied by diffuse consolidation on radiographic examination and BAL revealing a progressively bloodier return of the lavage fluid.

Bronchiolitis obliterans (BO) is defined by irreversible obstructive disease after BMT, often in the presence of chronic GVHD. This condition is accompanied by the presence of a normal or hyperinflated chest on plain radiography, or findings of patchy areas of "ground-glass" attenuation adjacent to areas of hyperinflation (mosaic attenuation) on high-resolution computed tomography.

\section{Management of patients with new pulmonary infiltrates}

Patients presenting with new-onset pulmonary infiltrates, with or without fever, were managed with consideration given to the presence of neutropenia and timing of pulmonary infiltrates (within 1 month, 2-4 months and $>4$ months after transplantation).

Febrile neutropenia (defined as a granulocyte cell count of $<0.5 \times 10^{9}$ cells $\cdot \mathrm{L}^{-1}$ ) was treated empirically with antibiotics: mezlocillin, cefazolin, and gentamicin. If the patient remained febrile after 5 days, amphotericin $\left(0.5 \mathrm{mg} \cdot \mathrm{kg}\right.$ body weight ${ }^{-1}$. day $^{-1}$ ) was added to the antibacterial therapy. If the patient's condition deteriorated, the antibiotic treatment was changed to amikacin and ceftazidime.

Empirical therapy for hospital-acquired pneumonia occurring after recovery of the neutrophil count was determined for each patient individually, based on previous culture results. Community-acquired pneumonia was initially treated with intravenous cefuroxime, and, in more severe cases, intravenous erythromycin or ciprofloxacin was added.

In patients with possible fungal infections, such as during febrile neutropenia or in the presence of nodular opacities on chest radiography, amphotericin was added to the treatment regimen.

\section{Bacteriological studies}

Sputum sampling was attempted in all patients with local infiltrates, and findings correlated with Gram staining results. Radiographic findings of pulmonary infiltrates were classified as local, diffuse or nodular. If patients with segmental or lobar shadows on chest radiography did not respond to empirical antibiotic therapy within $24-48 \mathrm{~h}$, fibreoptic bronchoscopy was performed in order to identify a possible opportunistic pathogen. In patients with bilateral diffuse infiltrates, fibreoptic bronchoscopy was performed at presentation to exclude DAH (when appropriate) or opportunistic infection. Patients presenting with nodular opacities underwent fibreoptic bronchoscopy or computed tomography-guided fine needle aspiration, depending on the size and location of the nodular lesion and provided they had a platelet count of $\geqslant 50,000$ cells $\cdot \mathrm{mL}^{-1}$.

\section{Data analysis}

Probability of survival analysis was carried out. The $95 \%$ confidence intervals (CIs) of the proportions and the fractional survival values were computed as 1.96 SEM in each direction. SEMs were calculated by the method of GREENWOOD [7].

Relevant literature was retrieved and reviewed using PubMed, with the most recent search performed on August 20, 2003, using the keywords stem cell/bone marrow transplantation and nonmyeloablative or dose reduced conditioning with, in turn, lung, pulmonary, cytomegalovirus, fungal infection, pneumonia, bronchiolitis obliterans and alveolar hemorrhage, using all alternative spellings and hyphenation.

\section{Results}

Fifty-three patients (37 male and 16 female) underwent NST between March 1996 and October 1998. The mean age of the recipients was 39 yrs (range 16-63 yrs). The median follow-up period for this cohort of patients was 28.5 months (range 1-91 months), and the last follow-up included in the study was performed on May 31, 2003.

During follow-up, $29(55 \%, 95 \%$ CI $40-68 \%)$ of the 53 patients died. A Kaplan-Meier survival curve is shown in figure 1 . Of the 53 patients, 35 survived beyond 100 days. Only two ( $7 \%$ of deaths, 95\% CI $1-23 \%$ ) patients died owing to IPS and respiratory failure. In nine $(31 \%, 95 \%$ CI $15-51 \%)$ patients, relapse of malignancy was the cause of death, whereas four $(14 \%, 95 \%$ CI $4-32 \%)$ patients died of sepsis (with adult respiratory distress syndrome in two patients) and one $(3 \%, 95 \%$ CI $0-18 \%)$ patient died of fungal pneumonia. Mortality at day 100 from transplant-related complications was $18.9 \%$ (95\% CI $9-32 \%$; 10 patients).

Most of the infectious complications occurred within 6 months after transplantation (fig. 2). Reviewing the microbial

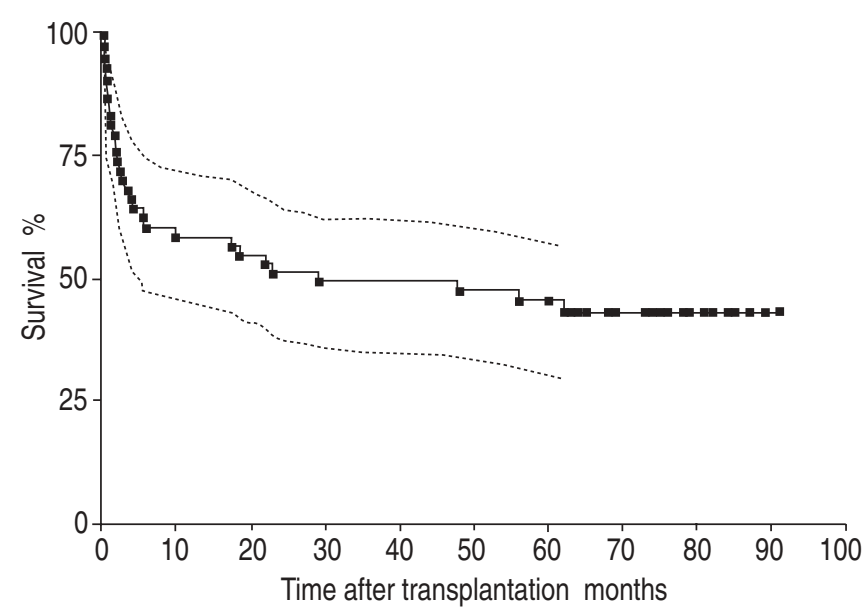

Fig. 1.-Kaplan-Meier survival curve (- survival; ……... 95\% confidence interval). The time at which each individual was censored is shown (ם). 
culture results obtained from these patients, 44\% (95\% CI $32-56 \%$ ) of positive cultures were from blood, and a further $47 \%$ (95\% CI 35-59\%) were from the upper respiratory tract (throat swab cultures and sinus secretions) and pulmonary secretions (reliable coughed sputum specimens and BAL samples). Four patients in this cohort developed bacterial pneumonia.

One patient developed $P$. carinii pneumonitis after discontinuing prophylaxis prematurely, and recovered after appropriate therapy. Two patients had Aspergillus pneumonia, which caused death in one patient, and, in the other, was associated with fatal sepsis. One diabetic patient developed pulmonary mucormycosis. This patient, detailed elsewhere [8], underwent pneumonectomy with complete eradication of the fungal infection, but died 6 weeks later due to chronic GVHD and liver failure.

CMV was not an evident cause of pneumonitis in any of the present patients. None of the BAL fluid or transbronchial biopsy specimens showed cytopathic changes typical of CMV. Additionally, blood and BAL fluid culture for CMV in patients suspected of CMV pneumonitis were negative. Using the polymerase chain reaction to monitor blood for the presence of CMV DNA, positive specimens were obtained over at least two consecutive weeks in 30 of the $46(65.2 \%$, 95\% CI 50-79\%) patients for whom consecutive test results were available. In the 12 patients who underwent fibreoptic bronchoscopy for various clinical indications, only one patient gave positive test results for CMV DNA in a BAL specimen, and this finding was associated with systemic CMV disease. However, blood specimens obtained simultaneously from that patient for CMV DNA analysis were negative.

The prevalence of GVHD at any time after transplantation was $51 \%(95 \%$ CI $37-56 \%)$ in the present group of patients. Nineteen per cent $(95 \%$ CI $8-32 \%)$ of the patients developed skin erythema with transient elevations of liver enzyme levels and were classified as GVHD grade I-II. A further 19\% (95\% CI 9-32\%) developed severe acute GVHD. Of the patients who survived $>100$ days after transplantation, $11 \%(95 \% \mathrm{CI}$ 4-23\%) developed severe extensive chronic GVHD, with death resulting from severe nonremitting GVHD in five $(9.4 \%, 95 \%$ CI $3-21 \%)$ patients.

Of the patients reviewed in the present study, $17(32 \%, 95 \%$ CI 20-46\%) developed pulmonary complications (table 2). There were only two $(3.8 \%, 95 \%$ CI $0-13 \%)$ patients in whom a diagnosis of IPS was made. One patient who had undergone transplantation because of non-Hodgkin's lymphoma
Table 2.-Pulmonary complications after nonmyeloablative stem cell transplantation ${ }^{\#}$

Pulmonary complication

Patients n (\%), 95\% CI

Bacterial pneumonia

Pneumocystis carinii pneumonitis

Cytomegalovirus pneumonititis

Fungal infection

Aspergillus

Mucormycosis

Pulmonary oedema

Adult respiratory distress syndrome

Idiopathic pneumonia syndrome

Pleural effusion

Drug-related pulmonary fibrosis

Total

4 (7.5), $2-18$

1 (1.9), 0-10

1 (1.9), 0-10

3 (5.7), 1-16

2 (3.8), 0-13

1 (1.9), 0-10

2 (3.8), 0-13

2 (3.8), 0-13

2 (3.8), 0-13

3 (5.7), 1-16

1 (1.9), 0-10

19

CI: confidence interval. ${ }^{\#}$ : in 17 of the 53 patients.

developed pulmonary infiltrates and respiratory failure 2 months after transplantation. Notably, this patient had received mediastinal radiotherapy as part of a previous treatment for lymphoma. The other patient, who had undergone transplantation for myelodysplastic syndrome, developed fever and bilateral diffuse pulmonary infiltrates 8 months after transplantation; however, there had been no previous exposure to irradiation.

Remarkably, none of the patients in the present cohort developed DAH. Additionally, despite the occurrence of chronic GVHD, post-transplant BO was not observed.

\section{Discussion}

Early reports noted significant pulmonary complications occurring in $40-60 \%$ of patients undergoing allogeneic BMT, and causing $30 \%$ of the mortality after such transplants [1, 9]. Since the incidence of pulmonary complications after autologous BMT is lower [10,11], it was thought that most of these complications were related to the immune reactions causing GVHD.

Strategies that significantly reduce the incidence of GVHD, such as the administration of T-cell-depleted bone marrow are associated with a reduced incidence of pulmonary complications, particularly IPS [6]. However, this method is associated with a high incidence of recurrence of haematopoietic

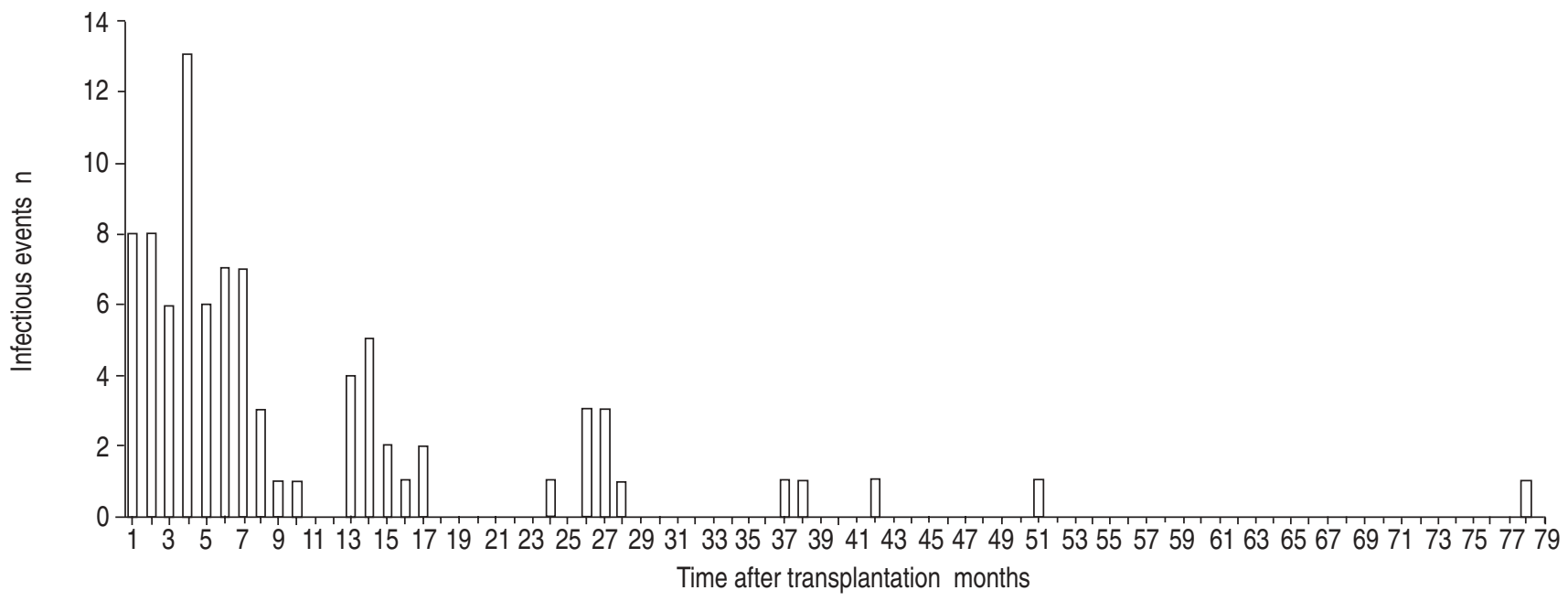

Fig. 2. - Timing of infectious events after nonmyeloablative stem cell transplantation. 
malignancy [12, 13], probably due to loss of the "graft-versusleukaemia" or graft-versus-tumour effect [14].

Nonmyeloablative conditioning is a less toxic irradiationfree approach, which results in a shortened and less pronounced period of pancytopenia while awaiting engraftment [4]. It is of note that none of the present patients received haematopoietic growth factors, thus making the results of the current study comparable with older studies describing transplant procedures undertaken before these growth factors were clinically available. In a previous report from the authors' institution, one-third of patients developed GVHD of varying degrees, and $15 \%$ eventually died due to complications of GVHD [4]. In a recently published paper describing dose-reduced conditioning in a series of 42 patients undergoing BMT for various haematopoietic malignancies, one-third of the patients developed acute GVHD, and GVHD was the cause of death in two patients [15]. Interestingly, in this same series, the incidence of graft failure was relatively high, which may have contributed to the decreased incidence of GVHD. Also, in the present study, it seems that patients had milder manifestations of GVHD, which were mainly related to allogeneic cell therapy. In other series of patients who underwent a similar protocol for chronic myeloid leukaemia, the incidence of acute GVHD was $11-35 \%[16,17]$, whereas the incidence of chronic GVHD was $66 \%$ [16].

Owing to the shortened nadir period and decreased severity of GVHD, nonmyeloablative reduced-dose conditioning is expected to lead to decreased incidence of opportunistic infections. The impact of morbidity related to such infections was decreased in the present patients relative to historical reports describing the outcome of allogeneic BMT. Several recently published studies still report a high incidence of CMV infection after nonmyeloablative conditioning [17-19], as noted in the present study; however, CMV disease was prevented, reflecting mainly infection surveillance and modern pre-emptive therapy. Invasive fungal infection was detected in two $(3.8 \%, 95 \%$ CI $0-13 \%)$ of the present patients, whereas the reported incidence of such infections is $10-23 \%$ $[19,20]$. It could be that the diagnosis of invasive fungal infection was missed in some of the present patients who died due to malignancy relapse or multiorgan failure as post mortem examinations were not performed in these patients.

None of the patients in the present series or in other cohorts [15-17] developed DAH, a complication that typically occurs early after BMT. The previously reported incidence of DAH after allogeneic and autologous BMT is similar (up to $20 \%$ ) [21]. This condition has also been described recently in patients who received T-cell-depleted bone marrow grafts [22]. The correlation of the appearance of DAH with the timing of neutrophil count recovery supports the hypothesis that neutrophils are strongly involved in the pathogenesis of DAH. The absence of DAH after nonmyeloablative conditioning is probably due to a short or absent nadir and a less dramatic recovery of peripheral blood counts, accompanied by the absence of severe mucositis and omission of total body irradiation.

The incidence of IPS in the present cohort was 3.8\%, compared to up to $20 \%$ in previous reports of allogeneic BMT $[1,2]$. KELEMEN et al. [16] also reported a low incidence of IPS $(5.5 \%)$ in their cohort of NST recipients. In other series of patients who underwent nonmyeloablative conditioning, there was no mention of IPS [15, 17]. Patients with IPS demonstrate diffuse lung injury following BMT with no infectious aetiology found [23]. Lung tissue specimens reveal interstitial oedema and inflammation or diffuse alveolar damage. IPS occurs more frequently in patients undergoing allogeneic BMT, with the intense conditioning that it entails, and is less common in HLA-identical sibling transplants for aplastic anaemia than in transplants for leukaemia [2, 23].
Observations from clinical studies suggest that total body irradiation [2, 24] and prior chest irradiation [11] are associated with a higher incidence of IPS. This is further supported by recent findings in a murine model of IPS, which showed that the combination of total body irradiation and allogeneic T-cells played an important role in the induction of lung injury [25]. A possible mechanism for this phenomenon is cellular damage caused by irradiation-induced free radicals and oxidants, with the affected cells becoming a target of activated allogeneic donor cells. Therefore, the decreased incidence of IPS after nonmyeloablative conditioning may be attributed to the absence of irradiation in the conditioning protocol and the decreased intensity of GVHD.

In the present series and those of BORNHAUSER et al. [15] and KELEMEN et al. [16], there were no patients with postBMT BO, although GVHD was still evident. Several factors, such as the occurrence of viral infections or inclusion of irradiation within the conditioning regimen, may be involved in inducing post-BMT BO. However, the single most important risk factor for the development of obstructive airway disease and BO after BMT is chronic GVHD [26]. Post-BMT BO is reported to occur in $4-10 \%$ of patients following allogeneic BMT $[27,28]$. There is only one report, describing two patients, of BO following autologous BMT [29]. There are no reports of patients developing BO following T-cell-depleted BMT. This suggests that post-BMT BO is alloimmune-mediated, and measures that reduce the intensity of GVHD reactions could prevent the occurrence of BO.

In conclusion, a low incidence of pulmonary complications after nonmyeloablative conditioning prior to stem cell transplantation was demonstrated. This may be partly attributed to a shortened nadir and accelerated bone marrow reconstitution, with decreased occurrence of infectious events. Diffuse alveolar haemorrhage was not observed in the present study, possibly because of nondramatic bone marrow reconstitution. The incidence of idiopathic pneumonia syndrome was low, probably related to the omission of irradiation from the conditioning protocol and decreased severity of graft-versus-host disease. Bronchiolitis obliterans was also not observed following bone marrow transplantation. The present study demonstrates that nonmyeloablative conditioning reduces pulmonary toxicity and pulmonary adverse effects, which are considered the Achilles heel of bone marrow transplantation.

\section{References}

1. Wingard JR, Mellits ED, Sostrin MB, et al. Interstitial pneumonitis after allogeneic bone marrow transplantation. Nine-year experience at a single institution. Medicine (Baltimore) 1988; 67: 175-186.

2. Weiner RS, Bortin MM, Gale RP, et al. Interstitial pneumonitis after bone marrow transplantation: assessment of risk factors. Ann Intern Med 1986; 104: 168-175.

3. Kantrow SP, Hackman RC, Boeckh M, Myerson D, Crawford SW. Idiopathic pneumonia syndrome: changing spectrum of lung injury after marrow transplantation. Transplantation 1997; 63: 1079-1086.

4. Slavin S, Nagler A, Naparstek E, et al. Nonmyeloablative stem cell transplantation and cell therapy as an alternative to conventional bone marrow transplantation with lethal cytoreduction for the treatment of malignant and nonmalignant hematologic diseases. Blood 1998; 91: 756-763.

5. Glucksberg H, Storb R, Fefer A, et al. Clinical manifestations of graft-versus-host disease in human recipients of marrow from HL-A-matched sibling donors. Transplantation 1974; 18: 295-304.

6. Breuer R, Or R, Lijovetzky G, et al. Interstitial pneumonitis 
in $\mathrm{T}$ cell-depleted bone marrow transplantation. Bone Marrow Transplant 1988; 3: 625-630.

7. Greenwood M. The natural duration of cancer. Rep Public Health Med Subj (Lond) 1926; 33: 1-26.

8. Glazer M, Nusair S, Breuer R, Lafair J, Sherman Y, Berkman N. The role of bronchoalveolar lavage in the diagnosis of pulmonary mucormycosis. Chest 2000; 117: 279-282.

9. Cordonnier C, Bernaudin JF, Bierling P, Huet Y, Vernant JP. Pulmonary complications occurring after allogeneic bone marrow transplantation. A study of 130 consecutive transplanted patients. Cancer 1986; 58: 1047-1054.

10. Wingard JR, Sostrin MB, Vriesendorp HM, et al. Interstitial pneumonitis following autologous bone marrow transplantation. Transplantation 1988; 46: 61-65.

11. Pecego R, Hill R, Appelbaum FR, et al. Interstitial pneumonitis following autologous bone marrow transplantation. Transplantation 1986; 42: 515-517.

12. Apperley JF, Jones L, Hale G, et al. Bone marrow transplantation for patients with chronic myeloid leukaemia: T-cell depletion with Campath-1 reduces the incidence of graft-versus-host disease but may increase the risk of leukaemic relapse. Bone Marrow Transplant 1986; 1: 53-66.

13. Butturini A, Gale RP. T cell depletion in bone marrow transplantation for leukemia: current results and future directions. Bone Marrow Transplant 1988; 3: 185-192.

14. Weiden PL, Flournoy N, Thomas ED, et al. Antileukemic effect of graft-versus-host disease in human recipients of allogeneic-marrow grafts. $N$ Engl J Med 1979; 300: 10681073.

15. Bornhauser M, Thiede C, Platzbecker U, et al. Dose-reduced conditioning and allogeneic hematopoietic stem cell transplantation from unrelated donors in 42 patients. Clin Cancer Res 2001; 7: 2254-2262.

16. Kelemen E, Masszi T, Remenyi P, Barta A, Paloczi K. Reduction in the frequency of transplant-related complications in patients with chronic myeloid leukemia undergoing BMT preconditioned with a new, non-myeloablative drug combination. Bone Marrow Transplant 1998; 21: 747-749.

17. Mohty M, Faucher C, Vey N, et al. High rate of secondary viral and bacterial infections in patients undergoing allogeneic bone marrow mini-transplantation. Bone Marrow Transplant 2000; 26: 251-255.

18. Nachbaur D, Larcher C, Kircher B, et al. Risk for cytomegalovirus infection following reduced intensity allogeneic stem cell transplantation. Ann Hematol 2003; 82: 621627.

19. Daly A, McAfee S, Dey B, et al. Nonmyeloablative bone marrow transplantation: infectious complications in 65 recipients of HLA-identical and mismatched transplants. Biol Blood Marrow Transplant 2003; 9: 373-382.

20. Hagen EA, Stern H, Porter D, et al. High rate of invasive fungal infections following nonmyeloablative allogeneic transplantation. Clin Infect Dis 2003; 36: 9-15.

21. Robbins RA, Linder J, Stahl MG, et al. Diffuse alveolar hemorrhage in autologous bone marrow transplant recipients. Am J Med 1989; 87: 511-518.

22. Ho VT, Weller E, Lee SJ, Alyea EP, Antin JH, Soiffer RJ. Prognostic factors for early severe pulmonary complications after hematopoietic stem cell transplantation. Biol Blood Marrow Transplant 2001; 7: 223-229.

23. Clark JG, Hansen JA, Hertz MI, Parkman R, Jensen L, Peavy HH. NHLBI workshop summary. Idiopathic pneumonia syndrome after bone marrow transplantation. Am Rev Respir Dis 1993; 147: 1601-1606.

24. Weshler Z, Breuer R, Or R, et al. Interstitial pneumonitis after total body irradiation: effect of partial lung shielding. Br J Haematol 1990; 74: 61-64.

25. Shankar G, Bryson JS, Jennings CD, Kaplan AM, Cohen DA. Idiopathic pneumonia syndrome after allogeneic bone marrow transplantation in mice: role of pretransplant radiation conditioning. Am J Respir Cell Mol Biol 1999; 20: $1116-1124$.

26. Clark JG, Schwartz DA, Flournoy N, Sullivan KM, Crawford SW, Thomas ED. Risk factors for airflow obstruction in recipients of bone marrow transplants. Ann Intern Med 1987; 107: 648-656.

27. Chan CK, Hyland RH, Hutcheon MA, Minden MD, Alexander MA, Kossakowka AE. Small airways disease in recipients of allogeneic bone marrow transplants: an analysis of 11 cases and a review of the literature. Medicine 1987; 66: 327-340.

28. Palmas A, Tefferi A, Myers JL, et al. Late-onset noninfectious pulmonary complications after allogeneic bone marrow transplantation. Br J Haematol 1998; 100: 680-687.

29. Paz HL, Crilley P, Patchefsky A, Schiffman RL, Brodsky I. Bronchiolitis obliterans after autologous bone marrow transplantation. Chest 1992; 101: 775-778. 